\title{
INTERPRETIVE SUMMARIES, OCTOBER 2012
}

Invited review: Milk production and reproductive performance: Modern interdisciplinary insights into an enduring axiom. By Bello et al., page 5461. The association between milk production and reproductive performance of dairy cows is generally believed to be antagonistic. In this article, we critically assess and discuss the limitations behind some of the evidence supporting this supposition. We then introduce interdisciplinary evidence based on modern statistical tools that address unique and previously ignored features of this association. We believe that the antagonism between milk production and reproductive performance is not necessarily universal and suggest better ways to study and even manage this association.

Fat-soluble vitamin (A, D, E, and $\beta$-carotene) contents from a Portuguese autochthonous cow breed-Minhota. By Ramalho et al., page 5476. In Portugal, commercial milk is obtained almost entirely from the Holstein breed. However, autochthonous breeds can also show dairy aptitude and produce milk of good quality. This is the first report investigating the effect of breed and seasonal factors in the vitamin contents of milk from Holstein and the autochthonous Portuguese breed Minhota. Breed had a pronounced effect on milk fat-soluble vitamins: the higher vitamin levels found in the milk from Minhota can be regarded as an important nutritional parameter that could increase the economic value of this breed.

Impact of curd milling on the chemical, functional, and rheological properties of starter-free Queso Fresco. By Van Hekken et al., page 552\%. Different curd milling treatments were evaluated to determine if this traditional step, which is time consuming and a potential source of bacterial contamination, is still needed in the manufacture of Queso Fresco (QF) made from pasteurized, homogenized milk. The chemical, functional, and textural properties of QF made incorporating a coarse, medium, or fine milling step and stored at $4^{\circ} \mathrm{C}$ for up to $8 \mathrm{wk}$ were compared with those of nonmilled QF. We found that the milling step disrupted the fragile matrix and resulted in a softer cheese that lost more whey during storage; sensory panelists could only detect a difference between the finest milled cheese and the other treatments.

Novel angiotensin I-converting enzyme inhibitory peptides produced in fermented milk by specific wild Lactococcus lactis strains. By Rodríguez-Figueroa et al., page 5536. Because diet has a role in the prevention and treatment of hypertension, research has focused on the manufacture of fermented dairy products with antihypertensive effects. In vitro studies suggested that fractions from milk fermented by specific wild Lactococcus lactis strains presented antihypertensive activity. In this study, we reported novel milk protein fragments associated with hypotensive activity.

Authentication of cow feeding and geographic origin on milk using visible and near infrared spectroscopy. By Coppa et al., page 5544. The ability of near infrared spectroscopy (NIRS), a rapid and low-cost technique, to trace cow feeding systems and farming altitude was tested on bulk milk. The NIRS correctly classified 95.5, 91.5, and $93.3 \%$ of samples comparing pasture with maize silage, hay, and fermented herbage feeding systems, respectively. In addition, NIRS correctly discriminated no-pasture from pasture milk, even in the diet with only $30 \%$ pasture $(5.4 \%$ cross-validation error). However, NIRS did not reliably trace milk geographic origin when the feeding system effect was isolated from the altitude effect. These findings may be exploited for the authentication of dairy products.

Reduction of ultraviolet light-induced DNA damage in human colon cancer cells treated with a lactoferrin-derived peptide. By Freiburghaus et al., page 5552. In several studies, milk consumption has been linked to improved health, with decreased risk of, for example, diabetes and colon cancer. However, the exact mechanisms of the specific milk components are not known. In this work, we investigated one milkderived component called lactoferricin 4-14. We have previously shown that this compound slows the growth of cells, and our hypothesis was that it might help repair processes in the cell. Our data may support the hypothesis that lactoferricin 4-14 has a role in repairing genetic damage, which may prevent cancer.

The stretchability of Mozzarella cheese evaluated by a temperature-controlled 3-prong hook test. By Ma et al., page 5561. We describe a novel and objective method for evaluating the stretchability of Mozzarella cheese with a tensile tester using a 3-prong hook. The stretching takes place in a temperature-controlled oil bath to ensure a constant temperature profile and to prevent moisture loss. In addition, a parameter from studies of polymer materials was used to evaluate stretchability and was found to apply very well to studies of cheese.

Steady shear rheological properties of micellar casein concentrates obtained by membrane filtration as a function of shear rate, concentration, and temperature. By Sauer et al., page 5569. The aim of this work was to generate information about the viscosity and flow behavior of micellar casein 
concentrates obtained by membrane separation as a function of shear rate, concentration, and temperature. The viscosity of the casein preparations increased exponentially with casein concentration and decreased with temperature. These dependencies were incorporated into a mathematical model that was able to accurately predict viscosity. This study will provide critical rheological data necessary for developing practical applications of micellar casein preparations.

Inactivation of Escherichia coli in milk and concentrated milk using pulsed-light treatment. By Miller et al., page 5597. Heat treatment is the standard method for the inactivation of microorganisms in the dairy industry. Recently, interest has increased in alternative technologies for microbial inactivation, including light-based treatments. Pulsed-light (PL) treatment consists of short, high-intensity pulses of broad-spectrum light emitted by gas discharge lamps, and it has been proven effective for the inactivation of microorganisms in a variety of foods or on food contact surfaces. The objective of this work was to evaluate the efficiency of PL on reducing Escherichia coli in milk and concentrated milk with varying concentrations of fat and solids, respectively.

Short communication: Isolation of a whey fraction rich in $\alpha$-lactalbumin from skim milk using tangential flow ultrafiltration. By Holland et al., page 5604. A permeate fraction enriched in $\alpha$-lactalbumin was produced from ultrafiltration of pasteurized skim milk using a plate-and-frame ultrafiltration system. The protein in the isolate was $>80 \%$ $\alpha$-lactalbumin. This permeate fraction could be used as a value-added ingredient for nutritional supplements.

Short communication: Within-day variation in fatty acid composition of milk from cows in an automatic milking system. By Larsen et al., page 5608. Milk samples of individual cows were taken from an automatic milk sampling system over $24 \mathrm{~h}$ and analyzed for the composition of fatty acids. The within-cow variation in content of individual fatty acids in milk fat was similar to the random variation, and we concluded that one random milk sample per cow is representative of fatty acid composition.

Effect of presynchronization with human chorionic gonadotropin or gonadotropin-releasing hormone 7 days before resynchronization of ovulation on fertility in lactating dairy cows. $B y$ Giordano et al., page 5612. Fertility of lactating dairy cows after synchronization of ovulation using a traditional Ovsynch protocol for second and subsequent artificial insemination is not optimal. Presynchronization with human chorionic gonadotropin (hCG) increased fertility by increasing the percentage of cows with their estrous cycle properly synchronized to the Resynch protocol, whereas presynchronization with gonadotropin-releasing hormone improved synchronization to the Resynch protocol but fertility was not improved when compared with non-presynchronized cows or cows presynchronized with hCG.

Association between stall surface and some animal welfare measurements in freestall dairy herds using recycled manure solids for bedding. By Husfeldt and Endres, page 5626. The objective of this observational study was to investigate the association between stall surface and some animal welfare measurements on dairy operations using recycled manure solids as a bedding material. Lameness prevalence was lower in deep-bedded freestalls (14.4\%) than in freestalls with mattresses (19.8\%). Severe lameness prevalence was also lower for cows housed in deep-bedded freestalls (3.6\%) than on mattresses $(5.9 \%)$. Similarly, the prevalences of hock lesions and severe hock lesions were lower in cows housed in deep-bedded freestalls than mattresses (49.4 vs. $67.3 \%$, and 6.4 vs. $13.2 \%$, respectively). Other welfare measurements were not associated with stall surface.

Herd characteristics and cow-level factors associated with Prototheca mastitis on dairy farms in Ontario, Canada. By Pieper et al., page 5635. Prototheca mastitis is an emerging disease in dairy cattle presenting as an incurable mastitis with high somatic cell counts and decreased milk yield. The aim of this case-control study was to identify risk factors for this disease. Farms that use extra-label infusion of an injectable drug into the udder, that use a high number of different injectable antibiotic products, that use any dry cow teat sealant, and that had more than 3 displaced abomasums within the last year were at greater risk for having Prototheca mastitis. Cows in the second or greater lactation and have high lactation-average somatic cell counts were at higher risk.

Effects of lactation and pregnancy on metabolic and hormonal responses and expression of selected conceptus and endometrial genes of Holstein dairy cattle. By Thompson et al., page 5645. Gene expression and protein synthesis in the fetal-maternal-placental unit must be coordinated for a successful pregnancy. The reduction in fertility of dairy cows caused by lactation could be mediated by alterations in key hormones and metabolites. Here, we found that lactation altered metabolic status, delayed initiation of cyclicity and lowered concentrations of progesterone in pregnant cows. Early expression of pregnancy-associated glycoprotein genes in the conceptus may be a key component necessary for a successful development of early pregnancy. 
Effects of lactation and pregnancy on gene expression of endometrium of Holstein cows at day 17 of the estrous cycle or pregnancy. $B y$ Cerri et al., page 565\%. Effects of lactation on uterine gene expression are largely unknown. At d 17 after ovulation, we found that expression of 702 genes was altered by pregnancy, and 210 genes were differentially expressed between lactating and nonlactating cows. The expression of 61 genes was affected by the interaction between pregnancy and lactation. Major biological functions affected by pregnancy were represented by immune-related genes and interferon-stimulated genes. Lactation affected genes related to immune system and developmental processes. Transcripts affected by lactation are candidate genes that could partly explain the subfertility in lactating dairy cows.

Herd-level association of serum metabolites in the transition period with disease, milk production, and early lactation reproductive performance. By Chapinal et al., page 5676. A total of 2,365 dairy cows from 55 freestall herds across North America had blood collected weekly to identify herd-level indicators, expressed as proportion of sampled animals with increased nonesterified fatty acids or $\beta$-hydroxybutyric acid or decreased calcium in wk -1 and wk +1 relative to calving that were associated with herd-level changes in the incidence of disease, milk production, and probability of pregnancy at the first insemination. Several herd-level thresholds were identified as predictors of herd performance.

Reproductive performance with an automated activity monitoring system versus a synchronized breeding program. By Neves et al., page 5683. This field study compared reproductive performance in 3 dairy herds over $1 \mathrm{yr}$, in which the primary reproduction management tool was an automated activity monitoring (AAM) system for heat detection for half the cows and a synchronization program for timed artificial insemination (TAI) for the other half. Time to pregnancy was not different or was shorter with the AAM system compared with a TAI-based program when 19 to $32 \%$ of total inseminations were based on observed estrus. The effect of management program depended on herd, with time to pregnancy shorter in 2 of 3 herds, but the differences between treatments were greater when considering only inseminations based on AAM or TAI (excluding cows with visually observed estrus).

Influence of social dominance on the secondary sex ratio and factors affecting hierarchy in Holstein dairy cows. By Hohenbrink and MeineckeTillman, page 5694. The ability to understand and influence the various factors that determine the sex of offspring in cattle would be a great benefit for the industry by making it possible to favor the birth of fe- male or male calves. The behavior of lactating Holstein cows was observed and their physical characteristics were determined to analyze whether these parameters are related to dominance status. We found that dominance status was correlated with body condition and the degree of lameness. Furthermore, dominance status had an effect on the sex ratio of calves, indicating that high-ranking animals have more female progeny.

Quality of bulk tank milk samples from Danish dairy herds based on real-time polymerase chain reaction identification of mastitis pathogens. By Katholm et al., page 5702. Real-time PCR-based testing for mastitis pathogens present in bulk tank milk can provide useful information for investigating possible causes of problems with milk quality. Our PCR results from bulk tank milk for Streptococcus agalactiae, Streptococcus dysgalactiae, and Streptococcus uberis were correlated with somatic cell count and total bacteria count. The PCR results for Staphylococcus aureus correlated with somatic cell count. A high quantity of the environmental mastitis pathogens Enterococcus spp. and Escherichia coli was positively correlated with total bacterial count.

Changes in glucose transporter expression in monocytes of periparturient dairy cows. By O'Boyle et al., page 5709. The transition period is characterized by dramatic changes in inflammatory cell functions that are associated with increased susceptibility to dairy cattle diseases. Compromised monocyte functions may be related to limited glucose uptake and utilization during the onset of lactation. The objective of this study was to determine how expression of monocyte glucose transporter (GLUT) changes during lactogenesis and in response to proinflammatory stimulation. Expression of GLUT was decreased postcalving, but was enhanced following endotoxin stimulation. Alterations in GLUT expression may influence monocyte inflammatory function during times of increased susceptibility to disease.

Characterization of the bovine innate immune response in milk somatic cells following intramammary infection with Streptococcus dysgalactiae subspecies dysgalactiae. By Beecher et al., page 5720. The bovine innate immune response to deliberate intramammary challenge with the pathogen Streptococcus dysgalactiae was found to be unique in terms of timing and immune targets induced. In particular, distinct expression patterns of the immune signals tumor necrosis factor- $\alpha$ and interleukin- $1 \beta$ were observed when compared with challenges with other mastitis pathogens. In addition, cows with different CXC chemokine receptor 1 (CXCR1) genotypes differed in levels of interleukin- 8 and CXCR1 gene signals in milk somatic cells. The difference in expression of 
these genes may influence the severity of disease within different genotypes. The results presented may provide information to develop novel therapeutic strategies for Streptococcus dysgalactiae udder infections.

Associations of dairy cow behavior, barn hygiene, cow hygiene, and risk of elevated somatic cell count. By DeVries et al., page 5730. The objective of this study was to determine the associations between dairy cow standing and lying behavior, barn cleanliness, cow hygiene, and the risk of experiencing elevated somatic cell count. Cow hygiene was affected by the standing and lying behavior of cows and by the cleanliness of the cow's environment. These results emphasize the need for cows to be provided clean standing and lying environments. Increasing the frequency of cleaning barn alley floors is an effective method for improving cow hygiene.

Bioeconomic modeling of intervention against clinical mastitis caused by contagious pathogens. By Halasa, page 5740. The epidemiologic and economic consequences of intervention against contagious clinical mastitis were investigated using a bioeconomic simulation model. Compared with short treatment duration, long duration and intensive antibiotic treatment of clinical mastitis without culling of unrecovered treated cows resulted in fewer clinical and subclinical mastitis cases and lower total annual costs of mastitis within a herd. Treatment regimens should be preceded by control strategies to reduce a high transmission rate of mastitis pathogens between cows to avoid large economic damage.

Effects of $\beta$-hydroxybutyrate and different calcium and potassium concentrations on the membrane potential and motility of abomasal smooth muscle cells in cattle. By Zurr and Leonhard-Marek, page 5750. An increase in plasma $\beta$-hydroxybutyrate (BHBA) above the reference range is known to increase the risk for dairy cows to develop left displacement of abomasum (LDA). Additionally, hypokalemia and hypocalcemia are observed in LDA cows. Causal relationships have not, however, been proven. The aim of the present study was to contribute to the understanding of the pathogenesis of LDA by characterizing the effects of BHBA, calcium, and potassium on slow waves and motility in abomasal smooth muscle cells.

Performance and metabolic responses of Holstein calves to supplemental chromium in colostrum and milk. By Ghorbani et al., page 5760. This study was designed to investigate the effects of chromium-L-methionine supplementation of colostrum and milk on performance and metabolic responses of newborn calves. Chromium did not affect dry matter intake, whereas it improved average daily gain, feed conversion ratio, and final body weight. In addition, blood $\beta$-hydroxybutyrate, nonesterified fatty acids, cholesterol, cortisol, and thyroxin were affected by chromium supplementation. Improved average daily gain and feed conversion ratio, along with changes in the measured metabolites and hormones, show the beneficial effects of colostrum and milk supplementation with chromium to improve performance and metabolic status of newborn calves.

Application of Wood's model to lactation curve of Italian Heavy Draft horse mares. By Centoducati et al., page 5770. Wood's model was applied to production of Italian Heavy Draft horse mare milk to evaluate and predict mare lactation curve. The correlation $\left(\mathrm{R}^{2}\right)$ values obtained confirm a good fitting process of lactation curve with Wood's model. The mares showed a higher milk yield than other breeds investigated in literature and the same qualitative profile.

Hyperinsulinemic clamp modulates milk fat globule lipid composition in goats. By ArgovArgaman et al., page 5776. The effects of nutrition and metabolic hormones on lipid composition of milk in goats are not well understood. The aim was to study the effect of insulin on lipid metabolism in the mammary gland and consequently milk lipid composition. High plasma insulin concentration reduced milk yield and fat concentration and elevated milk concentration of long-chain fatty acids. A high insulin concentration also tended to decrease milk triglyceride concentration and increase that of cholesterol and phospholipid. Milk lipid composition suggests preferred fatty acid channeling for membrane, rather than fat, synthesis by the mammary gland under elevated insulin plasma concentrations.

Alterations in milk and blood metabolomes during the first months of lactation in dairy cows. By Ilves et al., page 5788. Molecular composition of milk is influenced by lactation stage, in addition to genetic and environmental factors. In this study, massspectrometrical metabolomics was used to determine changes in milk and blood during the early stage of lactation. Little correlation was found between blood and milk metabolites, whereas in milk a clear common trend in changes was observed during the first months of lactation. Citrate and lactose had the greatest effect on these changes due to their abundance; however, the most significant decrease was in the levels of phosphorylated saccharides. The most significant changes in blood were associated with polyunsaturated phosphatidylcholines.

Metabolic and energy status during the dry period is crucial for the resumption of ovarian activity postpartum in dairy cows. By Castro et 
al., page 5804. The effect of energy and metabolic status in high-producing dairy cows on the resumption of ovarian cyclicity was examined from the dry period through early lactation. Cows with an earlier postpartum ovulation had greater energy and metabolic status throughout this period compared with cows without earlier postpartum ovulation. This was most apparent during the dry period. The hepatic factors investigated were related, in part, to the resumption of ovarian activity. Under good feeding management, ovarian function postpartum appears to be crucially influenced by energy status during the dry period.

Effects of methionine hydroxy copper supplementation on lactation performance, nutrient digestibility, and blood biochemical parameters in lactating cows. By Wang et al., page 5813. This experiment was conducted to determine whether replacing dietary copper sulfate with methionine hydroxy copper would improve the performance of dairy cows. Results in the current experiment were that both 50 and $100 \%$ replacement improved lactation performance, copper utilization, and the apparent digestibilities of acid detergent fiber and neutral detergent fiber compared with the control. Unexpectedly, $50 \%$ replacement was more effective than 100\% replacement in increasing apparent digestibilities of neutral detergent fiber and acid detergent fiber and in increasing blood serum copper concentration. Therefore, we suggest that replacing $50 \%$ dietary copper sulfate with methionine hydroxy copper is optimal for enhancing the performance of dairy cows.

Duodenal infusion of $\alpha$-linolenic acid affects fatty acid metabolism in the mammary gland of lactating dairy cows. By Yang et al., page 5821. Increasing the $\alpha$-linolenic acid content of milk fat might be desirable to enhance the nutritional value of milk. Our objective was to increase the concentration of 18:3 cis-9,cis-12,cis-15 in blood via duodenal infusion to study the effects of enhanced $\alpha$-linolenic acid on mammary metabolism of fatty acids in lactating dairy cows. We showed that increasing arterial concentration of 18:3n-3 affected uptake and synthesis of fatty acids in the mammary gland. These results may be of value when developing practical management strategies to enhance milk fat $\alpha$-linolenic acid and nutritional value of milk as a whole.

The logistic curve as a tool to describe the daily ruminal $\mathrm{pH}$ pattern and its link with milk fatty acids. By Colman et al., page 5845. Rumen $\mathrm{pH}$ is the main parameter to determine acidosis. A novel method to describe the daily ruminal $\mathrm{pH}$ pattern consists of fitting a logistic curve to continuous $\mathrm{pH}$ data points. As rumen $\mathrm{pH}$ changes are associated with changes in the milk fatty acid profile, the relationship between parameters of the logistic curve and milk fatty acids was assessed. Milk fatty acids did not change only with $\mathrm{pH}$ but also due to the variation in daily ruminal $\mathrm{pH}$.

The relationship between rumen acidosis resistance and expression of genes involved in regulation of intracellular $\mathrm{pH}$ and butyrate metabolism of ruminal epithelial cells in steers. $B y$ Schlau et al., page 5866. Previous research indicates that the extent of resistance to rumen acidosis is variable among ruminants fed a common diet. Acidosisresistant animals had lower total concentrations of volatile fatty acids (VFA), higher proportion of molar butyrate, and greater expression of sodium hydrogen exchanger isoform 3 (NHE3) in ruminal epithelial cells compared with acidosis-susceptible animals. Greater NHE3 expression in resistant animals might be attributed to a faster rate of simple diffusion of VFA in the undissociated form and subsequent dissociation in the ruminal epithelial cells.

Milk protein synthesis in response to the provision of an "ideal" amino acid profile at 2 levels of metabolizable protein supply in dairy cows. By Haque et al., page 5876. Supplying a well-balanced essential amino acid (AA) profile can help to decrease nitrogen waste from dairy cows by increasing the efficiency of protein utilization, permitting the reduction of protein intake. Providing an "ideal" AA profile (9 AA) at a low protein supply level increased the efficiency of protein utilization to the same degree as that at a high protein supply level. Balancing the AA profile with the 4 most deficient essential AA, more feasible in practice, increased the milk protein yield similar to an "ideal" profile.

Soybean meal substitution with a yeast-derived microbial protein source in dairy cow diets. $B y$ Sabbia et al., page 5888. The objective of this study was to examine the effects of substituting soybean meal with a yeast-derived microbial protein (YMP) in highproducing dairy cow diets. Ruminal ammonia nitrogen decreased with the inclusion of YMP, but we observed no other effects on rumen fermentation. Plasma glucose and $\beta$-hydroxybutyrate increased at higher YMP concentrations in the diet. Milk fat percentage and yield, total solids percentage and yield, energy-corrected milk, and fat-corrected milk showed a trend in diets that included YMP at 300 and 600 g. Substitution of soybean meal with YMP tended to increase total solids production and component-corrected milk in high-producing dairy cows that consumed high-forage diets.

Effect of adding a mycotoxin-sequestering agent on milk aflatoxin $M_{1}$ concentration and the performance and immune response of dairy cattle fed an aflatoxin $\mathbf{B}_{1}$-contaminated diet. By Queiroz 
et al., page 5901. This study examined the effect of adding 2 doses of a mycotoxin-binding clay adsorbent to diets contaminated with aflatoxin on transfer of the toxin to milk, immune response, and the performance of dairy cows. The high dose of the binder reduced the milk aflatoxin level compared with a diet containing the toxin with no binder, but the low dose did not. Both doses of the binder reduced the negative effects of aflatoxin on milk protein content and the immune response of cows. This study demonstrates that appropriate doses of certain mycotoxin adsorbents can be used to decrease negative effects of dietary mycotoxins on the safety of milk and dairy cow performance.

Maturation of mononuclear phagocytes in the lungs of young calves - In vitro study. By Batista et al., page 5909. The aim of this in vitro study was to identify the phases of increased susceptibility to respiratory diseases that affect calves in the first stage of life. For this, bronchoalveolar lavage samples from calves of 1 to 3 mo were collected and evaluated for cell functional activity. The respiratory system is not immunologically competent during the first 3 mo of life.

Effects of oil and natural or synthetic vitamin $\mathbf{E}$ on ruminal and milk fatty acid profiles in cows receiving a high-starch diet. By Zened et al., page 5916. Trans fatty acids produced during the ruminal metabolism of polyunsaturated fatty acids can affect cow milk fat content and human consumers' health. The trans-10 isomers (often produced in high quantities when cows receive high-starch, high-fat diets) especially, exhibited some negative effects. This study demonstrated that vitamin E supplementation of diets inducing a high proportion of trans-10 fatty acids in milk did not modify the ruminal metabolism of fatty acids and had only limited effects on milk fatty acids profile. Natural and synthetic vitamins had similar effects.

Disappearance of infused phytate from the large intestine of dairy heifers. By Ray et al., page 592\%. Accurate prediction of the availability of phosphorus to dairy cows might be limited by lack of knowledge of phytate digestion and absorption of phosphorus in the large intestine. Improved prediction of phosphorus availability from different feeds would help implement dietary strategies to reduce phosphorus excretion by dairy cows. This experiment with dairy heifers investigated phytate digestion and phosphorus absorption in the large intestine. Infused phytate was largely degraded in the large intestine; the inorganic phosphorus released disappeared partially from the large intestine. The results of this study may allow more accurate prediction of availability of phosphorus in different feeds.
Social behavior of young dairy calves housed with limited or full social contact with a peer. By Duve and Jensen, page 5936. Dairy calves are often separated from the dam at birth and housed individually. This study compared the effects of individual housing (limited social contact), pair housing from birth, and pair housing from 3 wk of age on the behavior of dairy calves. Pair-housed calves performed more social behaviors compared with calves housed individually, but only minor differences were found between calves pair-housed from birth and those pair-housed from 3 wk of age.

Effects of glucogenic and ketogenic feeding strategies on splanchnic glucose and amino acid metabolism in postpartum transition Holstein cows. By Larsen and Kristensen, page 5946. Glucogenic feeding was obtained with high allowance of sodium hydroxide-treated wheat grain in contrast to 2 ketogenic feedings obtained with high allowance of either fodder beets or alfalfa haylage in the early postpartum period. The high allowance of sodium hydroxide-treated wheat provided the most glucogenic feeding, as indicated by the greatest glucose release from the portal-drained viscera and the least recycling of lactate to the liver. Amino acid metabolism in splanchnic tissues seemed unaffected by amount of glucose absorbed from the small intestine. Apart from alanine, amino acids did not contribute substantially to liver gluconeogenesis in postpartum transition cows.

Effects of method of barley grain processing and source of supplemental dietary fat on duodenal nutrient flows, milk fatty acid profiles, and microbial protein synthesis in dairy cows. By Mutsvangwa et al., page 5961. Dietary composition and ruminal environmental conditions can alter the ruminal biohydrogenation pathways of dietary unsaturated fatty acids and, consequently, duodenal fatty acid flows and milk fatty acid composition. We investigated the interactive effects of barley grain processing (pelleting vs. dry-rolling) and source of supplemental fat (whole canola vs. flax) on duodenal fatty acid flow and milk fatty acid composition in dairy cows. Barley grain processing and source of fat altered ruminal fatty acid biohydrogenation, as reflected by changes in duodenal fatty acid flow and milk fatty acid composition.

Insertion depth of oral stomach tubes may affect the fermentation parameters of ruminal fluid collected in dairy cows. By Shen et al., page 5978. An oral stomach tube (OST) is a tool that can be used to collect ruminal fluid. Today, various types of OST are used in practice. However, opinions regarding the validity and representativeness of ruminal fluid collected via OST are controversial. Our study 
demonstrates that the controversy may be mainly attributable to the different insertion depths of the OST, which affects the representativeness of the ruminal fluid collected in dairy cows. To reduce saliva contamination, the OST should be inserted into the central rumen for ruminal fluid collection.

Use of Prevotella bryantii 25A and a commercial probiotic during subacute acidosis challenge in mid-lactation dairy cows. By Chiquette et al., page 5985. In this study, we evaluated a new bacterial strain (Prevotella bryantii 25A), which seemed promising as probiotic for dairy cows suffering from subacute acidosis, along with a commercial probiotic. The role of the commercial probiotic in regulating rumen $\mathrm{pH}$ was confirmed. Additionally, the probiotic seemed to prevent the decrease of one species of cellulolytic bacteria during subacute ruminal acidosis. Our results indicate that $P$. bryantii $25 \mathrm{~A}$ did not prove effective during subacute ruminal acidosis.

Effects of adding a concentrated pomegranateresidue extract to the ration of lactating cows on in vivo digestibility and profile of rumen bacterial population. By Jami et al., page 5996. In this study, we assessed the effect of concentrated pomegranate peel as a supplement to the daily nutrition of dairy cows. We assessed metabolic parameters such as milk yield, composition, and apparent health of the animals as well as the effect on the bacterial population residing in the digestive tract. We found an increase in digestion and milk yield, which was accompanied by changes in the microbial rumen communities observed.

Change of plasma volume, osmolality, and acidbase status in healthy calves after feeding of milk and water- and milk-based oral rehydration solutions. By Bachmann et al., page 6006. Although abomasal passage of oral rehydration solutions (ORS) prepared in milk is slower compared with that of ORS prepared in water, feeding these solutions resulted in equal rates of plasma expansion in healthy calves. Milk-based ORS increased the strong ion difference in plasma $2 \mathrm{~h}$ postprandial, an effect that might reduce metabolic acidosis in calves suffering from diarrhea. As milk feeding should be continued in diarrheic calves, preparing ORS in milk might be a simple method to treat diarrheal dehydration and acidosis.

Nutrient utilization, ruminal fermentation, microbial abundances, and milk yield and composition in dairy goats fed diets including tomato and cucumber waste fruits. By Romero-Huelva et al., page 6015. Ruminant production in the Mediterranean is limited by scarcity of pasture and poor quality, which makes waste fruits from greenhouse horticulture an interesting alternative to decrease the use of cereals in animal feeding. In this study, the replacement of $35 \%$ of cereal-based concentrate with feed blocks containing tomato and cucumber waste fruits reduced animal feeding cost and methane production, led to higher polyunsaturated fatty acid concentrations in milk, and did not promote significant changes in ruminal fermentation, nutrient utilization, or milk yield and quality in dairy goats.

A meta-analysis of the effects of feeding yeast culture produced by anaerobic fermentation of Saccharomyces cerevisiae on milk production of lactating dairy cows. By Poppy et al., page 602\%. A meta-analysis of a specific manufactured yeast culture was conducted on lactating dairy cows and stratified for sub-group analysis. The estimated increase in production performance for cows fed yeast culture in peer-reviewed studies was $1.18 \mathrm{~kg} / \mathrm{d}$ more milk, 1.61 $\mathrm{kg} / \mathrm{d}$ more $3.5 \%$ fat-corrected milk, or $1.65 \mathrm{~kg} / \mathrm{d}$ more energy-corrected milk. Dry matter was estimated to increase by $0.62 \mathrm{~kg} / \mathrm{d}$ in cows $<70$ days in milk and decreased by $0.78 \mathrm{~kg} / \mathrm{d}$ in cows later in lactation. This study demonstrates the benefits of feeding this manufactured yeast culture to dairy cows.

Rumen-protected lysine, methionine, and histidine increase milk protein yield in dairy cows fed a metabolizable protein-deficient diet. By Lee et al., page 6042. This experiment showed that cows fed protein-deficient diets have reduced nitrogen excretion in urine and manure, but may also have decreased milk yield if the diet is not supplemented with ruminally protected amino acids. We demonstrated that histidine, in addition to lysine and methinone, is a limiting amino acid in high-producing dairy cows fed diets based on corn silage and alfalfa haylage, for which microbial protein represents a large proportion of the metabolizable protein.

Using eastern gamagrass to construct diets that limit intake and caloric density for dairy replacement heifers. By Coblentz et al., page $605 \%$. Eastern gamagrass was harvested, ensiled, and subsequently blended into corn silage/alfalfa haylage diets for dairy replacement heifers, comprising 0, 9.1, 18.3, or $27.4 \%$ of the total dietary dry matter. The diet without eastern gamagrass (0\%) was offered for ad libitum and restricted ( $85 \%$ of ad libitum) intakes. Visually, eastern gamagrass haylage was not sorted by heifers and it reduced dry matter intake, as well as the caloric density of the blended diets. Inclusion of $27.4 \%$ eastern gamagrass, primarily as a substitute for corn silage, reduced average daily gain to $0.85 \mathrm{~kg} / \mathrm{d}$, which was comparable to performance on the restricted-intake diet $(0.88 \mathrm{~kg} / \mathrm{d})$; other diets produced average daily gain of 0.95 to $1.09 \mathrm{~kg} / \mathrm{d}$, which exceeded recommended weight gains for dairy heifers. 
Short communication: Grain-induced subacute ruminal acidosis is associated with the differential expression of insulin-like growth factorbinding proteins in rumen papillae of lactating dairy cattle. By Steele et al., page 6072. This study characterized the messenger RNA expression of genes involved in the insulin-like growth factor (IGF) axis in rumen papillae during the early onset of ruminal acidosis in lactating dairy cattle. Relative expression of IGF-binding proteins 3 and 5 were altered on the fourth day of feeding the high-grain diet. These results support the view that the IGF-1 axis is involved in the adaptation of the rumen epithelium to high-grain diets. Gaining insight into the mechanisms that control rumen epithelial adaptation to high-grain diets may provide opportunities to mitigate the detrimental effect that ruminal acidosis imposes on dairy cattle.

Technical note: Test of a low-cost and animalfriendly system for measuring methane emissions from dairy cows. By Hellwing et al., page 6077. Methane is a greenhouse gas, and cattle are major contributors to its global emission. Both national levels of emission and reduction strategies have to be verified to achieve international and governmental goals of reduction. Therefore, a low-cost chamber system was designed, constructed, and validated. We concluded that the system measures reliable and accurate methane emissions without compromising animal welfare.

Inbreeding depression on survival of Austrian Brown Swiss calves and heifers. By Fuerst-Waltl and Fuerst, page 6086. In this investigation, we analyzed the effect of inbreeding on mortality in Austrian Brown Swiss female calves and heifers. Frequencies of mortality were 3.2 and $9.3 \%$ during the first $30 \mathrm{~d}$ of life and until first calving, respectively. Inbreeding had a significant and unfavorable effect on the mortality of replacement stock. In the whole rearing period, heifer calves with an inbreeding of $10 \%$ had a $4.9 \%$ higher mortality rate compared with heifer calves with no inbreeding.

A recursive algorithm for decomposition and creation of the inverse of the genomic relationship matrix. By Faux et al., page 6093. As the number of genotyped animals increases, some genomic prediction models have issues related to inversion of the genomic relationship and related matrices. We developed a recursive algorithm to approximate the inverses of those matrices. The algorithm converges after a few rounds of recursion, but additional work is needed to reduce computing costs further.

Improved accuracy of genomic prediction for dry matter intake of dairy cattle from combined European and Australian data sets. By de
Haas et al., page 6103. In this study, we demonstrated that using dry matter intake (DMI) phenotypes from multiple countries increases the accuracy of genomic breeding values for this important trait, provided a multi-trait approach is used. Data from Australia, the United Kingdom, and the Netherlands were combined to estimate the accuracy of genomic prediction for DMI using single- and multi-trait models. The highest accuracies were estimated for all countries when data were analyzed with a trivariate model (with DMI a different trait in each country), with increases of up to $5.5 \%$.

Genetic correlations of days open with production traits and contents in milk of major fatty acids predicted by mid-infrared spectrometry. By Bastin et al., page 6113. Optimal fertility is vital for profitable dairy production systems. Indirect selection using highly heritable traits recorded earlier in lactation can enhance the accuracy of selection and thus genetic gain. Therefore, the objective of this study was to investigate the genetic relationships between fertility and contents in milk of the major fatty acids predicted by mid-infrared spectrometry. Results substantiated the known relationship between body fat mobilization in early lactation and fertility and indicated an opportunity to use fatty acid content in milk to supplement genetic predictions of fertility.

Comparative genomics between fly, mouse, and cattle identifies genes associated with sire conception rate. By Li et al., page 6122. The objective of this study was to investigate the association of highly conserved spermatogenesis genes and POU1F1 pathway genes with sire conception rate in cattle. The spermatogenesis genes $M A P 1 B$ and PPP1R11 and the POU1F1 genes (STAT5A and POU1F1) showed significant associations with bull fertility. Moreover, MAP1B was associated with female fertility traits.

Validation of RNA isolated from milk fat globules to profile mammary epithelial cell expression during lactation and transcriptional response to a bacterial infection. By Brenaut et al., page 6130 . Mastitis, an inflammation of the mammary gland, is the most costly infectious disease of dairy ruminants worldwide. The early steps of the response of mammary epithelial cells (MEC) to mastitis remain poorly defined. Here, we report a noninvasive method using milk fat globules (MFG) as a source of mammary RNA to follow the dynamics of the global transcriptional response of MEC during the course of a bacterial infection. This method provides an easily accessible source of RNA that reflects MEC transcriptional activity.

Genetic variation for infection status as determined by a specific antibody response against Mycobacterium avium subspecies paratubercu- 
losis in milk of Dutch dairy goats. By van Hulzen et al., page 6145 . Control strategies based on management restrictions to reduce transmission, culling of infected goats, and vaccination have not been able to eradicate Johne's disease from infected herds. Selective breeding for lower susceptibility may be a useful additional tool to contribute to control of Johne's disease. This study provides information on genetic variation for infection status and shows that selective breeding can contribute to a more effective control of Johne's disease in goats.

Evaluation of propane flaming for reducing bacterial counts in sand bedding. By Hogan et al., page 6152. The use of propane flaming of recycled sand reduces mastitis pathogen populations in bedding. Onfarm recycling of bedding by separation of sand from manure reduces bedding costs and aids the environmental sustainability of farms. However, the subsequent use of sand reclaimed from passive water-settling ponds or mechanical separation often results in increased exposure of cows to pathogens. We determined that the use of propane flaming was a clean and rapid antibacterial procedure for sanitizing recycled sand.
The effect of reproductive performance on the dairy cattle herd value assessed by integrating a daily dynamic programming model with a daily Markov chain model. By Kalantari and Cabrera, page 6160 . Profitability of a dairy herd is closely associated with reproductive performance and replacement decisions. Optimization and simulation models are useful methods to evaluate the effect of these factors on farm profitability. This study found a positive relationship between reproductive performance and herd value under optimal replacement decisions, as well as a strong interaction among milk productivity, reproductive performance, and herd value.

Association of the chemical composition of the total mixed ration with milk, fat, and protein yield lactation curves at the individual level. By Caccamo et al., page 6171. Lactation curves for milk and milk components in dairy cattle show variation in peak and persistency of yield, which is partially explained by dietary composition and feed management. This study indicated a confounding response that can be obtained when multiple nutrients are not taken into account simultaneously. 